\title{
Der Brückenbauer. Intervista con Dario Siddi
}

\section{Marie Usadel $^{1} \&$ Francesco Corgiolu $^{2}$}

\author{
${ }^{1}$ Ruhr University Bochum
}

${ }^{2}$ ARCI Il Calderone APS

\section{Introduction (English)}

Dario Siddi was a teacher and a student, theater director and actor, an archaeologist. Born in 1944 he lived and worked as a middle school teacher in Sant'Antioco and Calasetta in Sardinia. In 1988 he founded Il Calderone, a small theater company: however, over time the association would also become active in other areas of social and cultural life in Sant'Antioco, from the integration of immigrants to the organization of cinematic events. After his passing in 2020 his friends called him a bridge-builder, which is probably the best description of the role he played in the archaeological life of Sant'Antioco.

Together with his fellow members of Il Calderone he initiated a cooperation with researchers of the Ruhr-University Bochum (Germany). The resulting archaeological project, under the direction of Constance von Rüden, is aimed at investigating the prehistoric settlement system of the Canai-plain in the southern part of Sant'Antioco with a particular interest in the Nuragic period. Since 2017 there have been annual field campaigns comprising a systematic survey in the plain, targeted prospection of the surrounding Nuragic sites as well as the detailed documentation of the area called Gruttiacqua in the south-western part of the Canai-plain. In 2019 it was possible to begin excavating part of the Nuragic village of Gruttiacqua. Since this project represents one of the rare cases where the archaeologists were chosen by the community and not the other way around, the cooperation between students, international researchers and members of Il Calderone is an essential, original element that directly and more effectively involves the local community.

The following interview was conducted in September 2018 by Maja Gori and Antonia Davidovic-Walter, as part of a larger series of interviews, to elaborate on the ideas about cultural heritage as well as expectations and motivations of the local community regarding the archaeological fieldwork. As a citizen of Sant'Antioco and trained archaeologist Dario Siddi was able to communicate possibilities and future perspectives, and to build an ideal bridge to bring the local community closer to foreign archaeologists and more generally to scientific research and vice versa. 
We want to take this opportunity to share his story and his words as an example on how a collaboration between the involved and interested public and international researchers can originate and develop, following Marshall's account of community archaeology, "not because it is politically correct, but because it enriches our discipline." (Marshall 2002: 218).

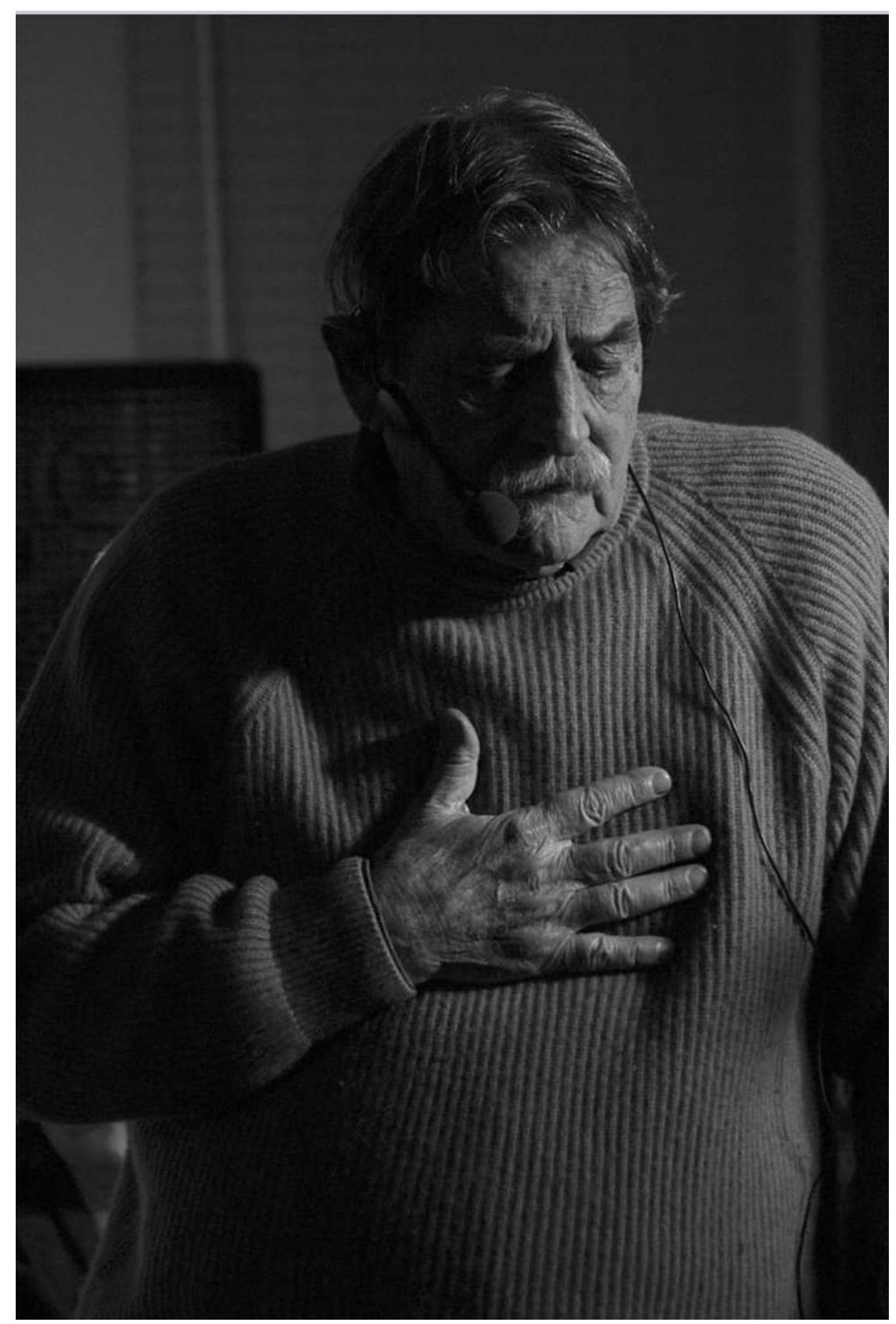

Figure 1. Dario Siddi on stage (Photo by Davide Guala).

\section{Introduzione (italiano)}

Dario Siddi è stato un insegnante e uno studente, regista teatrale e attore, un archeologo. Nato nel 1944 ha vissuto e lavorato come insegnante nelle scuole medie di Sant'Antioco 
e Calasetta in Sardegna. Nel 1988 fonda Il Calderone, una piccola compagnia teatrale. Tuttavia, nel tempo, l'Associazione diventerà attiva anche in altri settori della vita sociale e culturale di Sant'Antioco, dall'integrazione degli immigrati all'organizzazione di eventi cinematografici.

Dopo la sua scomparsa nel 2020 alcuni amici lo hanno definito un costruttore di ponti, probabilmente la migliore definizione per il suo impegno pubblico di divulgatore in ambito archeologico.

Insieme ad altri soci de Il Calderone, Dario ha avviato una collaborazione con i ricercatori della Ruhr-University Bochum (Germania). Il progetto archeologico che ne è scaturito, sotto la direzione di Constance von Rüden, è volto ad indagare il sistema insediativo preistorico della piana di Canai nella parte meridionale di Sant'Antioco con particolare interesse per il periodo nuragico. Dal 2017 sono state eseguite campagne annuali sul campo che hanno compreso un'indagine sistematica nella pianura, una prospezione mirata dei siti nuragici circostanti, nonché la documentazione dettagliata dell'area denominata Gruttiacqua nella parte sud-occidentale della piana Canai. Nel 2019 è stato possibile iniziare lo scavo di una parte del villaggio nuragico di Gruttiacqua. Poiché questo progetto rappresenta uno dei rari casi in cui gli archeologi sono stati scelti dalla comunità e non il contrario, la cooperazione tra studenti, ricercatori internazionali e Il Calderone rappresenta un elemento essenziale, originale e coinvolge direttamente e più efficacemente la comunità locale.

La seguente intervista è stata condotta nel settembre 2018 da Maja Gori e Antonia Davidovic-Walter, come parte di una serie di interviste fatte per ragionare sull'idea di patrimonio culturale, così come sulle aspettative e le motivazioni della comunità locale riguardo al lavoro archeologico sul campo. Da abitante e cittadino di Sant'Antioco e da archeologo qualificato, nelle sue risposte Dario Siddi è stato capace di condensare possibilità e prospettive future, e di costruire un ponte ideale per avvicinare la comunità locale agli archeologi stranieri e più in generale alla ricerca scientifica e viceversa.

Vogliamo cogliere questa opportunità per condividere la sua storia e le sue parole: un esempio di come una collaborazione tra un pubblico partecipe e interessato e un team di ricercatori internazionali possa nascere e svilupparsi seguendo la lezione di Marshall sull'archeologia comunitaria, "not because it is politically correct, but because it enriches our discipline." (Marshall 2002: 218). 


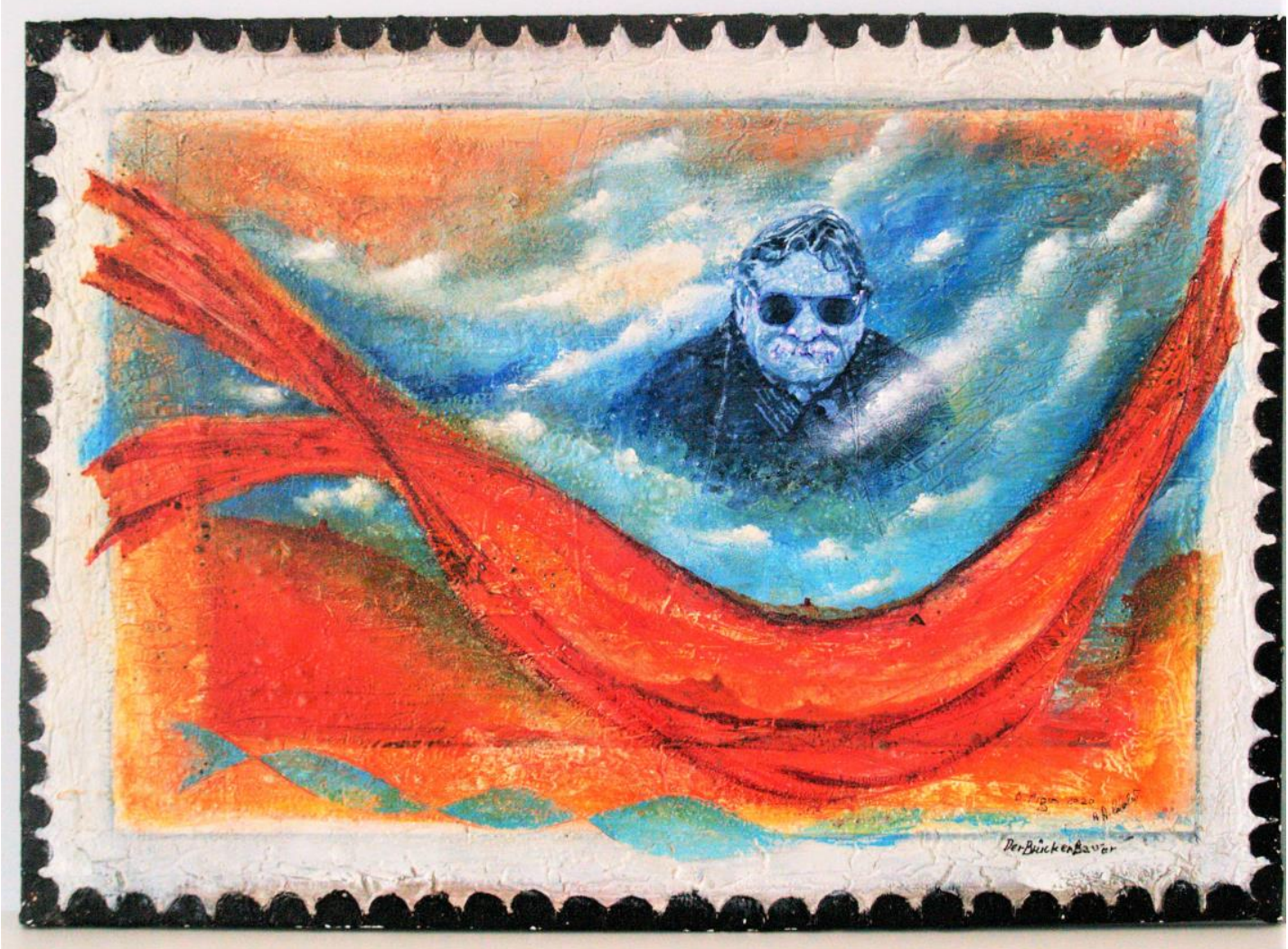

Figure 2. Der Brückenbauer, dipinto di A. A. Calabró e B. Piga (2020).

\section{Intervista (italiano)}

Maja Gori: La prima domanda riguarda Il Calderone e questo progetto. Com'è nata l'idea di un progetto archeologico?

Dario Siddi: Il Calderone nasce come compagnia teatrale e per tanto tempo si è dedicato solamente a questa attività, immagino che qualcuno l'avrà già detto. Dopo una pausa di quattro, cinque anni, ha ripreso con il teatro, ma in tempi più recenti Il Calderone ha ampliato il proprio campo di intervento fino a quello che è adesso, cioè un'associazione culturale e di promozione sociale con tutto una serie di obiettivi e di campi di intervento che sono comunque previsti nel nostro statuto. Com'è sorta l'idea? Io insegnavo alla scuola media: dopo che sono andato in pensione, mi sono riscritto all'università e ho ripreso una vecchia passione: l'archeologia. Mi sono dato tutta la trafila degli esami, laureandomi in un corso di laurea magistrale. A questo punto ho pensato di trasferire le mie conoscenze a chi fosse disponibile ad ascoltare, soprattutto a quelle persone che si interessano o guardano al periodo nuragico come all'età delle origini. Noi veniamo da lì, in effetti noi veniamo anche da lì, ma veniamo da tante altre parti, anche. Considerando il fatto che tanti appassionati purtroppo si interessano alle fantasie che leggono nelle reti di comunicazione e che quindi c'erano tante cose da sfatare, tanti concetti da lasciar perdere, abbiamo pensato di affrontare la materia in modo serio, in modo documentato, in modo particolare. Il Calderone in quel momento si apre: "Hai voglia di parlare di nuragico? Ti 
piace, ti interessa? Vieni!'. In quella fase, l'archeologia va ad aggiungersi agli altri campi di intervento del Calderone.

Maja Gori: Il Calderone appunto: come mai questo nome?

Dario Siddi: Calderone perché, quando lo fondammo e, per fare tutte le cose in regola, andammo dal notaio, eravamo una serie di individui molto diversi: ecco il calderone, cioè un recipiente comune per tante anime, ne più e ne meno come adesso. Insomma, siamo diversi, a volte ci scontriamo anche, ovviamente: se non ci si scontra non è bello. [Ride]

Maja Gori: Per quale motivo preferite questo arco cronologico? Perché proprio il periodo nuragico e non quello prima o quello dopo?

Dario Siddi: Perché, per quanto riguarda la storia antica della Sardegna, molte persone sono più affascinate dal periodo nuragico per il via delle sue manifestazioni, spesso in modo fuorviante. Ad esempio, il nuraghe: una costruzione alta, ben caratterizzata, non ce ne sono uguali al mondo, siamo i primi, i più importanti. Nuragic über alles. Diamoci una calmata e vediamo questa civiltà in contesti mediterranei o europei e capiamo che non è esattamente così. Per questo abbiamo cominciato con queste lezioni: inizialmente con pochissimi elementi, pian piano la cosa ha cominciato a prendere piede e adesso tutto sommato questa attività funziona. A quel punto ne ho approfittato e ne ho approfitto tutt'ora, quando capita, per far capire che prima della civiltà nuragica ci sono 4000 anni nei quali la Sardegna è ben rappresentata e ben caratterizzata con le sue diverse culture: insomma, con pazienza, si tratta di intervenire su un terreno vergine, che è fatto di molte opinioni, idealizzate e false piuttosto che non basate su fatti concreti.

Maja Gori: Oltre all'interesse personale, come presidente e fondatore dell'associazione, che ruolo hai avuto nel convogliare gli altri verso l'archeologia?

Dario Siddi: [Ride] Recentemente ci siamo ricordati i primi incontri con tre, quattro, cinque elementi che venivano per la curiosità di conoscere il nuragico, però poi si comportavano come quegli allievi un po' indisciplinati. Inizialmente si trattò di fare uno sforzo, parlare per 10 minuti ma parlare in modo serio, insomma. Invece, da quando la cerchia degli studenti, chiamiamoli così, si è allargata, si autoregolamentano. Adesso c'è una buona partecipazione, o quantomeno la curiosità...e dall'altra parte la curiosità è la prima molla. Poi fai filtrare quelli che sono i contenuti reali.

Maja Gori: In genere, a parte l'aspetto scientifico, cosa interessa del progetto?

Dario Siddi: A me interessa in modo particolare: considero questo contatto con gli altri la vera divulgazione, perché ne sentiamo di tutti i colori, insomma. Invece, visti anche gli aggiornamenti della ricerca, i nuovi ritrovamenti ci mettono di fronte ad una società complessa di cui conosciamo assolutamente poco, visto che era una società senza lingua, cioè senza scrittura meglio, non senza lingua...e quindi difficilissima poi da analizzare nelle classi sociali. Si ipotizza "organizzazione sociale", si ipotizza "gerarchia degli 
insediamenti", si ipotizza "erano cantoni": si vabbè, c'era forse una federazione di cantoni, forse. E quindi si brancola un po': tante motivazioni sfuggono, tanti aspetti dei rituali sfuggono, non abbiamo un nome, non abbiamo una divinità rappresentata. A meno che diversi bronzi nuragici non rappresentino delle divinità, ma su questo si discute. Abbiamo i templi dedicati a un culto dell'acqua, va bene, perché ce li abbiamo, si vedono, si possono indagare e si indagano in effetti: l'acqua e poi? Il clero? Non sappiamo niente del clero. Eppure, noi abbiamo i santuari nuragici, ma qual era il rapporto con il clero? Qual era il rapporto fra l'élite, visto che si parla di élite nuragiche, e il clero? I santuari, che cosa sono? Sono centri di aggregazione? Sì, è vero, ma anche di distribuzione delle risorse? Può darsi. Luoghi dove si manifesta la potenza dell'élite? Anche, ma poi tutto il resto sfugge.

Maja Gori: Si, ho capito. Diciamo da questo punto di vista riportare l'aspetto scientifico nella società è centrale?

Dario Siddi: Certo, assolutamente. Altrimenti parliamo d'altro. Diciamo che i nuraghi son stati costruiti dai marziani e va bene, no? Va bene... [Ride]

Maja Gori: Da un punto di vista personale, che rapporto hai con la regione e con il nuraghe e con lo scavo, con il sito?

Dario Siddi: Per quanto riguarda lo scavo su siti nuragici, non ne ho. Io sono arrivato all'archeologia alla veneranda età di settantaquattro anni. Mi sono laureato nel 2015, figurati se qualcuno mi faceva scavare. Però ho avuto esperienze di scavo in due tombe puniche, qui nella nostra necropoli. Ho assistito alle campagne di scavo anche perché ho avuto l'onore e il privilegio di stringere amicizia con un grande dell'archeologia sarda che purtroppo è morto: si chiamava Paolo Bernardini, lui mi faceva partecipare ai suoi scavi. L'esperienza di scavo è un'esperienza assolutamente straordinaria, almeno per quel poco che ho visto e che ho vissuto. Aprire una tomba inviolata, chiusa 5000 anni fa, insomma una bella esperienza, fa venire i brividi.

Maja Gori: E con questo scavo?

Dario Siddi: Questo scavo e questa esperienza, è un po' uno dei sogni. È nata così, perché per caso incontrammo Thomas e Constance e fra una battuta e una risata e l'altra: "Perché non vieni a scavare qui?" E loro videro, visitarono il sito e lo trovarono ricco di potenzialità. E per fortuna l'università di Bochum ha risposto positivamente. Quello che dobbiamo riuscire a fare adesso è convincere le nostre amministrazioni e le nostre università di questa potenzialità. Basta pensare che in un'isola come questa, una piccola isola, piccola per modo di dire, però relativamente piccola, a fronte di 40, più o meno, nuraghi, quindi con una densità di una certa rilevanza, non ce n'è uno "tirato" fuori. E noi abbiamo questo splendido sito che ci fa intravedere una continuità di vita e di frequentazione molto ampia, e non solo lì ma anche nei dintorni: questo è un momento splendido per andare avanti. D'altra parte, cerchiamo di convincere le nostre amministrazioni che l'archeologia non è quella delle vetrine di un museo, è anche quello, ma se proprio la vogliamo mettere sul pratico, l'archeologia è pane, perché da posti di 
lavoro: oltre all'enorme interesse scientifico ci sono anche questi aspetti che non sono trascurabili. I centri che sono andati in quella direzione, vedi Barumini, ripeto oltre all'interesse scientifico, danno da lavorare a un sacco di gente. $\mathrm{O}$ si capisce questo, oppure perdiamo un treno.

Maja Gori: Quindi i nuraghi sono un simbolo della Sardegna: si può dire che sono un simbolo nazionale sardo?

Dario Siddi: Un simbolo d'identità, questo sì. Chi sa perché ci si ritrova. Per quella, c'è molto la bandiera dei Quattro Mori. Però quando tu metti un nuraghetto, come ad esempio fa un nostro amico che quando sta dipingendo, ogni tanto, in qualche modo inserisce nei suoi quadri un nuraghe: chissà perché gli viene da farlo? Perché lo sente. E quindi il nuraghe è un emblema, certo.

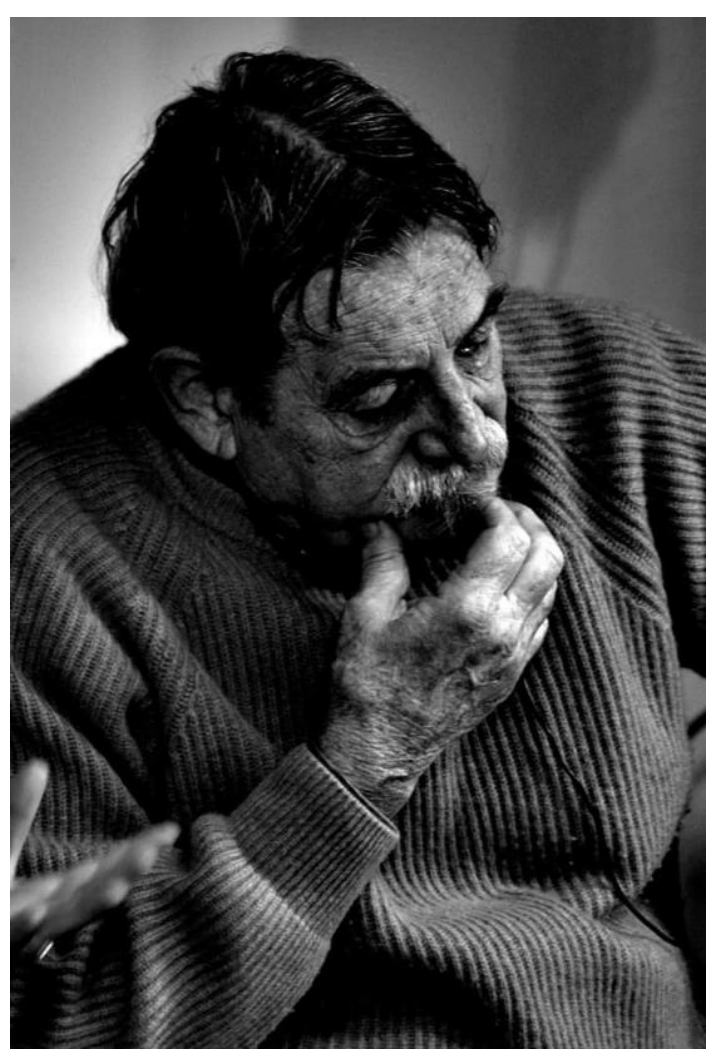

Figure 3. Dario Siddi (Photo by Davide Guala).
Maja Gori: Quindi è un simbolo identitario forte?

Dario Siddi: Fortissimo. Tant'è vero che la cosa straordinaria è che, quando i nuraghi nel periodo del bronzo finale non vengono più costruiti, si continua a riprodurli nel bronzo, nella pietra, nella steatite, in tutti i modi, in tutti i modi. E quindi, questo antico simbolo, resiste.

Maja Gori: Prima che iniziasse questo progetto, cosa conoscevi, cosa ti interessava del sito nel quale sta lavorando adesso l'università di Bochum? Perché proprio quello?

Dario Siddi: Sai, del sito si conosce abbastanza poco. Si sapeva di questo nuraghe, che poi alla fine i nuraghi lì sono due: perché c'è un nuraghe arcaico, cioè un nuraghe a corridoio, che poi viene sviluppato e diventa un nuraghe complesso. Si conoscevano anche alcuni basamenti di capanne. Ti dico questo perché, quando per la prima laurea, tantissimo tempo fa, preparavo l'esame in una materia, in una disciplina che non si chiamava Archeologia ma Antichità Sarde, che io diedi con uno dei padri dell'archeologia sarda cioè Lilliu, portai all'esame delle foto con dei fondi di capanne, lui disse "Eh, Gruttiacqua, quanto mi piacerebbe tornarci" e io gli risposi "Professore venga", "Eh, vediamo, vediamo...": non venne mai. Comunque, la cosa che affascina di quel sito è intanto la posizione. E poi questa complessità, la presenza di quelle capanne, la presenza di quello che comunemente a Sant'Antioco viene chiamato il laghetto di Gruttiacqua e che con tutta probabilità era 
una riserva idrica per quell'insediamento. La presenza di un pozzo sacro e, a poche centinaia di metri da questo sito, una tomba di giganti, l'unica cosa di nuragico che è stata scavata, qui a Sant'Antioco. Una tomba di giganti. Per questo, quella zona ha sempre suscitato un certo fascino e la curiosità di sapere.

Maja Gori: Quindi posso dire che per te l'epoca nuragica è più interessante delle epoche successive o precedenti?

Dario Siddi: Ł̀ una bella gara. A me interessa, certo. Ma anche nel periodo immediatamente successivo, nell'età del ferro, l'incontro con i fenici, con l'elemento fenicio, è di una enorme complessità e fascino. D'altra parte, noi siamo qui a Sulky e sono i fenici che fondano Sulky, in un periodo in cui sul meno stava sorgendo Roma, un pochino dopo stavano sorgendo le città etrusche: quindi noi siamo all'interno di una delle prime città d'Italia, di centri urbani, non chiamiamola città. Ecco, questo momento di transizione, l'incontro fra due etnie che non danno segni e che non portano segni di conflittualità, anzi, l'elemento fenicio che viene accettato dalla popolazione locale e il tofet, che è un'istituzione prettamente fenicia, lo sta a dimostrare. Perché all'interno del tuo tofet accogli dei vasi che sono nuragici? Cosa è successo? Vuol dire che ci sono stati matrimoni misti, vuol dire che c'è stato l'incontro. E oggi ne abbiamo bisogno d'incontri, o no?

Maja Gori: Assolutamente, sì.

Maja Gori: Cosa ti è arrivato da parte dell'università di questo progetto? Che comunicazione c'è stata? Che contatto hai avuto con gli archeologi, diciamo proprio a livello di informazioni?

Dario Siddi: Ma, per quanto riguarda il progetto, da parte dell'università niente. Praticamente abbiamo scarsi contatti con l'università. Sarebbe estremamente importante e auspicabile che l'università, se non altro quella di Cagliari, visto che è la più vicina, si unisse a questo progetto. D'altra parte, anche Constance si sta dando da fare come università di Bochum con l'amministrazione comunale di Sant'Antioco e il povero Calderone che fa da tramite e che cerca fondi. Noi quest'anno abbiamo ottenuto un grosso contributo dalla Fondazione di Sardegna, mentre il Comune ha fatto la sua parte, né più né meno nella quantità dell'anno scorso. Si tratta davvero di convincere le istituzioni a partecipare a questo progetto, perché più si allarga la cerchia, più partner ci sono e meglio è. Non dico che ci debba essere una pletora di collaborazioni, però quantomeno la partecipazione delle forze e delle istituzioni culturali: poi i finanziamenti arrivano, perché quando il lavoro comincia a prender piede, tu finanzi quest'anno, finanzi l'anno venturo e presenti un progetto con la partecipazione di un équipe internazionale, diventa qualcosa che rimane e che ha un suo proseguimento nel tempo.

Maja Gori: Che tipo di comunicazione ti piacerebbe avere dagli archeologi, nel senso pensi che ci sia un modo per comunicare questa campagna di scavo? 
Dario Siddi: Sì. Io credo, visto che queste campagne sono possibili anche grazie all'intervento di fondi pubblici, che bisogna rendere conto alla comunità. L'anno scorso non è stato fatto, anche perché abbiamo avuto problemi con alcuni proprietari delle aree del sito. Giustamente però l'amministrazione comunale lo chiede ed io credo che sia giusto. E anche Constance è d'accordo. Quest'anno bisogna fornire una informativa su quello che si fa, che si è fatto e su quello che si intende fare. Anche perché la popolazione locale è molto vicina a quel sito, lo ama: una conferenza, un incontro pubblico serve a stimolare e a creare un'alleanza con la popolazione. A Sant'Antioco, nelle belle giornate di primavera, chi non si fa la passeggiata al laghetto di Gruttiacqua? Chi? La gente ama quel luogo e se vengono mostrate le sue potenzialità, scientifiche e culturali, economiche, allora a quel punto la gente fa pressione, l'opinione pubblica fa pressione. Quindi è fondamentale l'informazione.

Maja Gori: Cosa ti piacerebbe vedere realizzato, oltre a queste conferenze? Che tipo di comunicazione? Che tipo di iniziativa vorresti che partisse?

Dario Siddi: Intanto una comunicazione, nel senso che tu davvero fai vedere quello che hai fatto finora e parli di prospettiva, però. Quindi indirizzare parte anche la conferenza verso quell'aspetto. "Tirare" fuori un sito di questo tipo significa lavorarci 20 anni insomma: voi ci sarete io no. Voi vedrete, forse e spero, la realizzazione di questo mio, nostro sogno. Cercatemi, sarò da qualche parte!

Un'altra cosa, quando l'equipe dell'università di Bochum avrà più dati a disposizione, sarebbe molto interessante presentarlo magari ad un convegno o con una pubblicazione scientifica. In effetti adesso sono in una fase assolutamente preliminare. Adesso vedi tante cose, ma fino a quando non cominci a usare la piccozza, fino a quando non scavi... allora vedremo cosa faranno i proprietari.

Maja Gori: L'ultima domanda: oltre al fattore culturale, questo progetto ha anche finalità sociali, nel senso di stimolare il turismo, la crescita culturale?

Dario Siddi: Questo progetto ha infatti diversi aspetti. Quello scientifico, culturale e, per quanto riguarda il grosso pubblico, avere a disposizione un sito scavato, voglio dire visitabile, sai cosa vuol dire? Entri all'interno di correnti di turismo culturale o di altro genere. Vuoi che qualcuno non metta lì una biglietteria? Vuoi che qualcuno non metta lì il chioschetto per le bibite? Vuoi che, come è successo negli altri siti, sorge una cooperativa di gestione del sito? Ecco che cosa vuol dire, come si allarga: una volta esplorati e messi a disposizione della comunità, è l'indotto e quello che gli fiorisce attorno. Oltre alla crescita culturale per la popolazione stessa, questo è evidente insomma, anche perché vai lì e gli dici: guarda dove vivevano, guarda e non farti idee strane.

\section{References}

MARSHALL Y. 2002. What is Community Archaeology? World Archaeology 34.2: 211-219. 
